\title{
Narrow-linewidth multi-wavelength random distributed feedback laser
}

\author{
Daniel Leandro, Sergio Rota-Rodrigo, Diego Ardanaz and Manuel Lopez-Amo, Senior Member, \\ IEEE.
}

\begin{abstract}
In this work, narrow-band emission lines are generated by means of two random distributed feedback fiber laser schemes. Spectral line-widths as narrow as 3.2 pm have been measured, which significantly improves previous reported results. The laser is analyzed with the aim of obtaining a spectral line-width as narrow as possible. Additionally a variation of this setup for multi-wavelength operation is also validated. Both schemes present a simple topology that use a combination of phase-shifted fiber Bragg gratings and regular fiber Bragg gratings as filtering elements.
\end{abstract}

Index Terms- fiber laser, distributed feedback laser, random laser, stimulated Raman scattering, Rayleigh scattering.

\section{INTRODUCTION}

$\mathrm{S}_{\mathrm{n}}^{\mathrm{I}}$ NCE the first laser emission in a mirror-less active medium was demonstrated in 1966 by Letokhov [1], random lasers have not been thoroughly studied until the recent years. This increasing interest relies on the singular properties of random lasers when compared to conventional lasers based on periodic structures [2].

In particular, a random fiber laser based on the amplified distributed feedback (DFB) along a single-mode fiber (SMF) was recently proposed by Turitsyn et al. [3]. In this laser, the feedback is provided by the weak backscattered light due to Rayleigh scattering along tens of kilometers of SMF and the amplification is given by the stimulated Raman scattering effect. This laser generates stable stimulated light emission in a spectral band of a few nanometers by using a simple all-fibre configuration. Following this study, a number of works have been published exploiting the properties of random DFB fiber lasers based on Raman scattering, such as their lower noise figure when compared to other Raman pumping schemes for telecommunication applications [4,5] or its flat response when tuned by means of a tunable filter [6]. Additionally, modulated random DFB fiber lasers overcome the locking frequency

Manuscript received March 31, 2015. This work was supported by the Spanish Government projects TEC2013-47264-C2-2-R and INNOCAMPUS.

D. Leandro, S. Rota, D. Ardanaz and M. Lopez-Amo, are with Department of Electric and Electronic Engineering, Public University of Navarra, Campus Arrosadia S/N, 31006, Navarra, Spain. (e-mail: daniel.leandro@unavarra.es).

Copyright (c) 2015 IEEE. Personal use of this material is permitted. However, permission to use this material for any other purposes must be obtained from the IEEE by sending a request to pubspermissions@ieee.org. problems of the conventional fiber laser configurations [7]. Other works have demonstrated the flat wavelength response when tuned cascaded operation at higher Stokes components [8,9] and have also addressed the efficiency issue [10]. Sensing is also a promising field of application of random DFB lasers with a number of works published on this subject during the last years [11-14].

Together with the broadband gain and high stability, the absence of mode competition and mode hopping in Raman scattering based random lasers due to its mode-less behavior [15] is of special interest in the design of multi-wavelength lasers. Numerous works have been published in this regard, using Sagnac interferometers [16,17], Fabry Perot filters (FPF) [18,19], a Lyot filter [20] or a long-period fiber gratings-based Mach-Zehnder interferometer combined with a FFP [21] to select the lasing wavelengths. Another approach published in [22] uses a programmable filter to attain a fully switchable 18wavelength laser.

Typically, non-filtered random DFB fiber lasers based on Raman scattering have a line-width of several nanometers which exceed the requirements of many practical applications. Recently a few studies have been published in this respect, striving for narrow emission lines by using different spectral filters. A FPF is used in [19] to obtain a tunable multiwavelength random DFB fiber laser with a line-width as narrow as $40 \mathrm{pm}$ using a hybrid erbium-Raman gain. In the same manner a FPF is also utilized in [18] to realize a multiwavelength laser. Moreover, a fiber Bragg grating is used to achieve a line-width of $50 \mathrm{pm}$. Finally a tunable multiwavelength random DFB laser is proposed in [21] with a linewidth down to $34 \mathrm{pm}$ by using a combination of a FPF and an interferometer based on long period gratings. A good review about DFB random lasers can be found in [23].

In this work, a random DFB fiber laser with a line-width of $3.2 \mathrm{pm}$ is presented, which significantly improves any other result as far as the authors know. The proposed topology is a simple all-fiber setup that uses a combination of fiber Bragg gratings as the spectrum filters. In addition, a variation of the setup for multi-wavelength operation is also presented.

\section{EXPERIMENTAL SETUP}

The laser topology presented is based on a forward-pumped random distributed feedback (DFB) fiber laser. A wavelength division multiplexer (WDM) is used to inject the pump laser (IPG RLD 3-1445) into the cavity formed by $50 \mathrm{~km}$ of 
standard single mode fiber (SMF). On one side, the SMF serves as the active medium for the gain generation due to the stimulated Raman scattering effect. On the other side, the SMF acts as a distributed mirror, providing a weak feedback along the fiber because of the Rayleigh scattering. The scattered light travels back into the port 2 of an optical circulator which role, together with other components, is to filter the light and redirect it into the cavity. The design of this filtering mirror is the key point of the study, since depending on the setup particularly narrow band emission lines can be attained. Accordingly, in this study two different topologies are presented.

\section{A. Single line emission.}

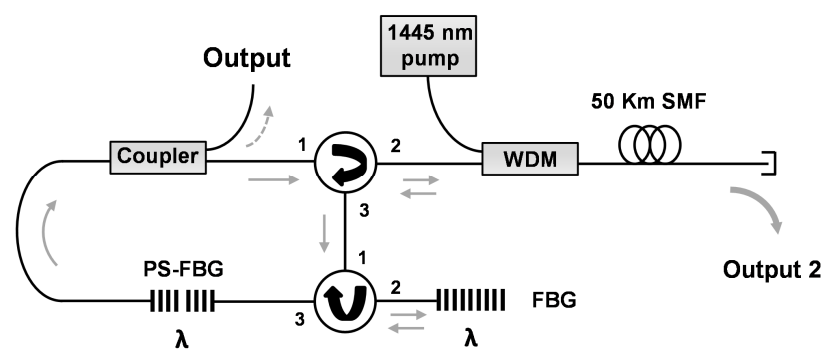

Fig. 1. Setup of the single-line random distributed feedback fiber laser.

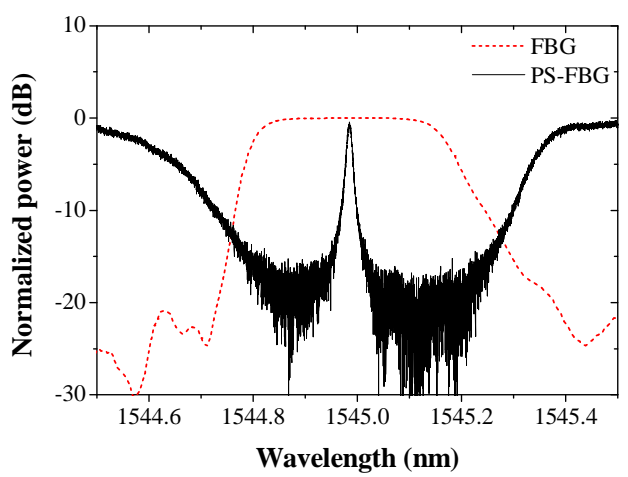

Fig. 2. Reflected and transmitted spectra of the tunable FBG and PS-FBG respectively.

The first proposed setup can be seen in Fig. 1. The main purpose of the system is to obtain a random laser by using the narrow central transmission peak of a phase shifted fiber Bragg grating (PS-FBG). The full width at half maximum (FWHM) of the central transmission peak is $11 \mathrm{pm}$. To select the central peak of the PS-FBG, the light is initially filtered by a fiber Bragg grating (FBG) centered at $\lambda=1545 \mathrm{~nm}(\mathrm{FWHM}=$ $0.35 \mathrm{~nm}$ ) which is properly connected to an optical circulator that allows the FBG to operate in reflection. The next component placed in the loop is a PS-FBG centered at $\lambda$ in a serial configuration which function is to narrow the emission line. In this manner, the resulting transmitted line of the loop is the combination of the reflected spectrum of the FBG and the PS-FBG response in transmission as depicted in Fig. 2. Finally, an optical coupler is included in the loop mirror in order to extract part of the signal and use it as the main output port. Additionally, an alternative output will be taken from the far end of the fiber (output 2 in Fig.1).

\section{B. Multi line emission.}

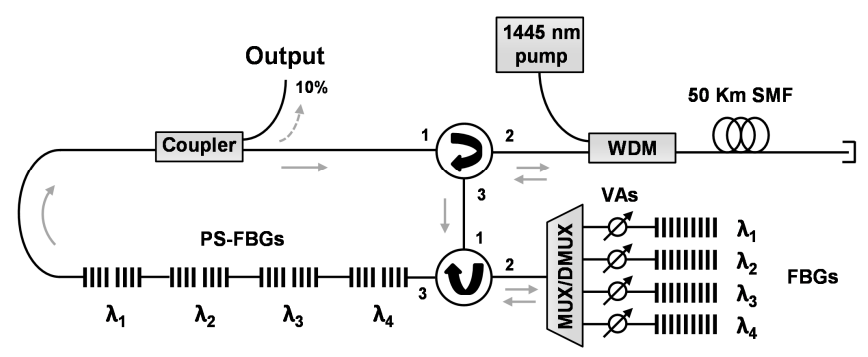

Fig. 3. Setup of the multi-wavelength random distributed feedback fiber laser.

A second scheme is also presented with the aim of increasing the number of emission lines. In this regard, four pairs of PS-FBGs and FBGs corresponding to the wavelengths $\lambda_{1}=1539.8 \mathrm{~nm}, \lambda_{2}=1545 \mathrm{~nm}$, at $\lambda_{3}=1549.6 \mathrm{~nm}$ and $\lambda_{4}=1552.9$ $\mathrm{nm}$ are utilized. The FWHM of the central transmission peaks are 14.5, 11, 19.2 and $29.9 \mathrm{pm}$ for the PS-FBGs placed at $\lambda_{1}$, $\lambda_{2}, \lambda_{3}$ and $\lambda_{4}$ respectively. A multiplexer/demultiplexer (MUX/ DEMUX) is used as can be seen in Fig. 3. The MUX/DMUX has four $200 \mathrm{GHz}$-wide channels (ITU channels C31, C35, C41 and C47) with insertion losses of $1.8-2.2 \mathrm{~dB}$ per channel. This device permits the inclusion of a variable attenuator (VA) in every branch to independently control the losses of each channel, adding the lowest possible losses to the cavity as reported in [24]. In this case, the PS-FBGs are concatenated in a serial configuration. It is worth saying that in order to avoid undesired reflections every fiber end of both setups was immersed in refractive index matching gel.

\section{RESULTS AND DISCUSSION}

\section{A. Single line emission.}

In order to investigate the performance of the proposed setups, a series of experiments have been carried out. Initially, a single emission line was analyzed by means of a Brillouin optical spectrum analyzer (BOSA by Aragon Photonics) with a resolution of $0.08 \mathrm{pm}$. As can be seen in Fig. 4, the narrowest emission line obtained has a quasi-lorentzian shape with a FWHM of $3.2 \mathrm{pm}$, which is more than ten times narrower than other reported random lasers as far as the authors know $[18,19,21]$. This emission line corresponds to a pump power of $1.42 \mathrm{~W}$ and a coupling ratio of $70 / 30$ with a $30 \%$ of the power re-inserted into the cavity. It can be also noted in Fig. 4 that the stimulated Brillouin scattering has a suppression ratio higher than $40 \mathrm{~dB}$ for that configuration. The first anti-Stokes line arises after increasing the pump power; reaching a suppression ratio close to $35 \mathrm{~dB}$ for a pump power increment between 70-110 $\mathrm{mW}$ depending on the setup. 


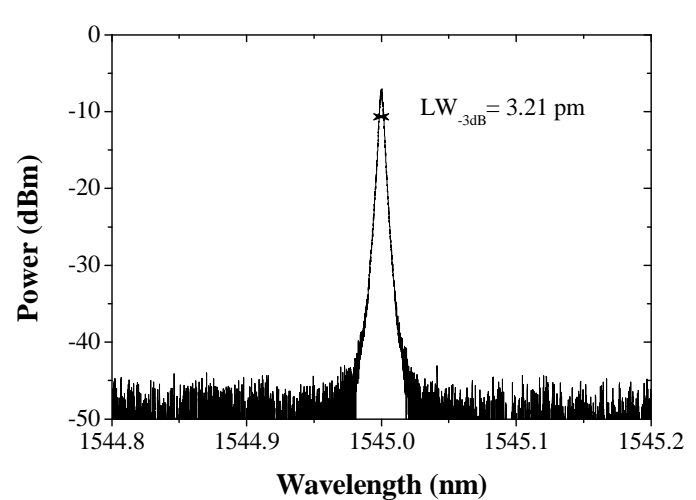

Fig. 4. Optical spectrum of the generated emission line.

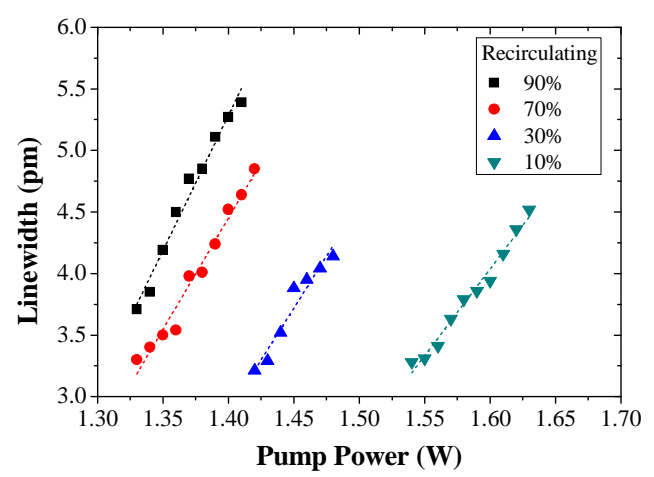

Fig. 5. Line-width variation with the Raman pump power for different coupling ratios.

The dependence of the line-width on the pump power has been also studied. With the aim of finding the optimal coupling ratio of the optical coupler placed in the loop, the experiment was repeated for different coupling values (Fig. 5). The results show that there is a clear increase of the line-width with the pump power as previously reported in $[6,18,25,26]$, obtaining the narrowest possible line-width right above the lasing threshold in every case. Furthermore, the increase rate of the line-width with the pump power varies with the coupling ratio. After applying a linear fitting, the gradient increases from 14 to $22 \mathrm{pm} / \mathrm{W}$ as the re-injected power into the cavity increases from $10 \%$ to $90 \%$. The selected coupling ratio also implies a trade-off between lasing threshold and peak power as can be seen in Fig. 6. The maximum peak power achieved is $1.2 \mathrm{dBm}$ which corresponds to the case of a $10 \%$ of re-inserted power with a pump power of $1.67 \mathrm{~W}$. Since the output port of the laser is placed inside the loop mirror, the attained peak power values are smaller than in other random lasers but are close to $0 \mathrm{dBm}$ when the extracted power from the loop mirror goes beyond the $50 \%$.

It is worth noticing that the results depicted in Fig. 6 also prove that the studied emission line is a laser itself, clearly showing the lasing threshold for each different coupling ratio. As it has been reported in studies before [3], one of the main properties of the random lasers based on Raman scattering is its longitudinal mode-less behavior due to the distributed reflection of the light along the fiber because of the Rayleigh scattering. In order to demonstrate the full random operation of the proposed laser, a test showing this mode-less operation was carried out. In this experiment, the output light was received by means of a photo-detector (Agilent $83440 \mathrm{~B}$ ) and studied in an electrical spectrum analyzer (Rohde \& Schwarz FSP30) with a resolution as good as $1 \mathrm{~Hz}$. The self-beating signal of the emission line can be clearly seen in Fig. 7 with different spans $(6 \mathrm{GHz}, 90 \mathrm{kHz}$ and $10 \mathrm{kHz})$ and resolution bandwidths $(100 \mathrm{kHz}, 300 \mathrm{~Hz}$ and $100 \mathrm{~Hz}$ respectively). There is no evidence of longitudinal mode beating, thus the random laser operation is verified.

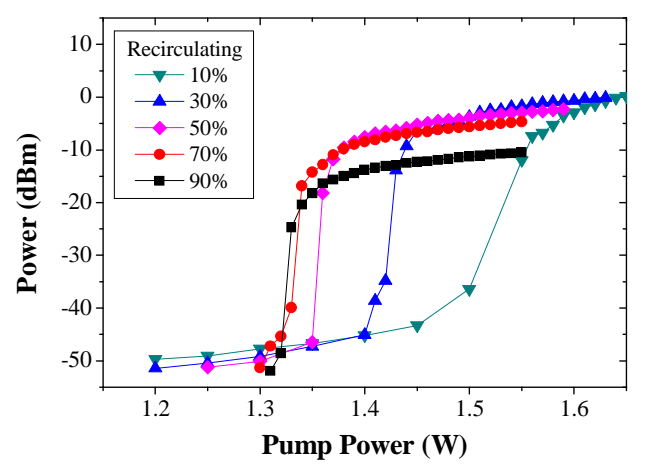

Fig. 6. Peak power of the emission line vs pump power for different coupling ratios.

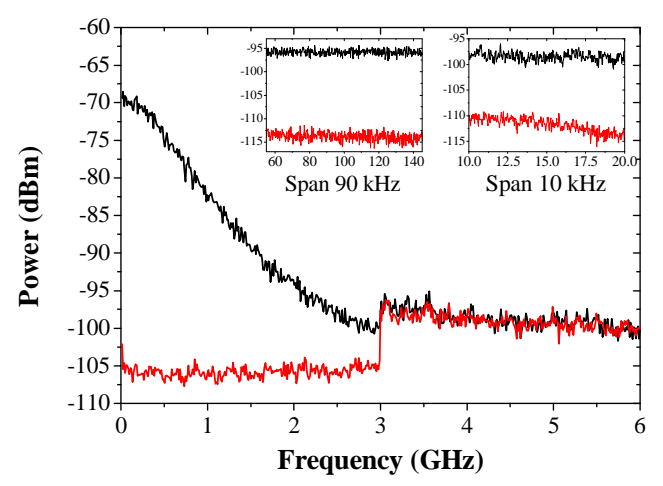

Fig. 7. Laser's electrical self-beating signal for different spans/resolutions (black) and the photo-receiver's noise level (red).

Another experiment was performed with the aim of comparing the emission lines obtained at the main port (loop mirror) with the measured at the output 2 (in Fig. 1). In order to avoid reflections and to extract the pump power remaining at output 2 , an isolator was placed followed by a WDM before the measuring device. Additionally, a variable attenuator was used to avoid any damage to the equipment. Figure 8 shows the spectrum measured at the output 2. The FWHM was in the best case (right above the lasing threshold) $6.45 \mathrm{pm}$, two times broader than in the best case obtained at the output placed in the loop mirror. Since the main part of the power travels codirectionally with the pump wave, the peak power measured is higher, reaching around $14 \mathrm{dBm}$. On the other hand, the optical signal to noise ratio (OSNR) is $22 \mathrm{~dB}$, about $18 \mathrm{~dB}$ smaller than the obtained in the loop mirror. Therefore, the 
output used could vary depending on the application: the one placed in the loop mirror provides narrower emission lines with higher OSNR but the output 2 offers higher peak power.

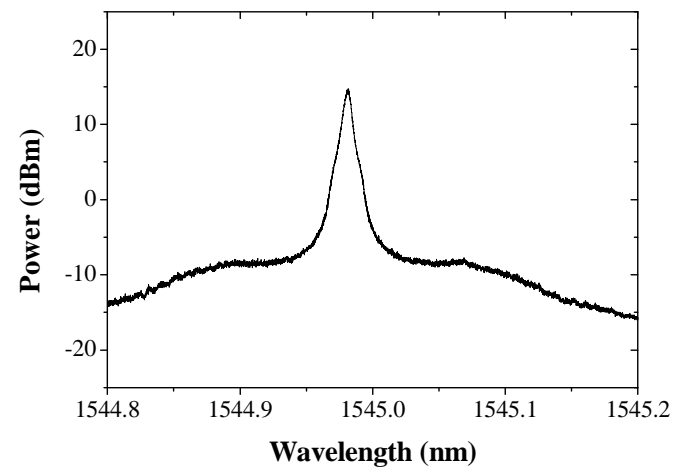

Fig.8. Optical spectrum of the generated emission line at the output 2.

Finally, a last test was carried out to evaluate the differences between performing the filtering process inside or outside the laser's cavity. In this regard, the tunable and the phase-shifted fiber Bragg gratings were removed from the loop and placed at the output port (using a 3-port circulator) as depicted in Fig. 9. The resulting emission line was measured using the BOSA, obtaining a FWHM of $12 \mathrm{pm}$ that is essentially equal to the FWHM of the PS-FBG. The peak power measured for this emission line is $-28 \mathrm{dBm}, 21 \mathrm{~dB}$ lower than in the previous case. Additionally, the OSNR measured is close to $20 \mathrm{~dB}$, which is $20 \mathrm{~dB}$ smaller than the obtained in the case in which the filters are located inside the loop. In order to compare both emission lines, the normalized spectra measured can be seen in Fig. 10. These results clearly evidence that placing the filters inside the loop greatly improves the performance of the laser in terms of line-width, peak power and OSNR.

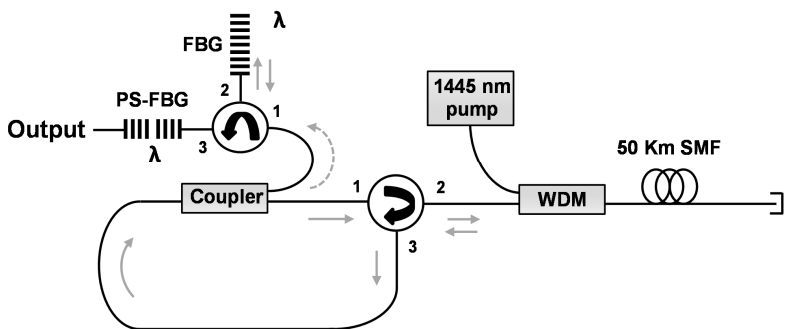

Fig. 9. Experimental setup where the filtering process is done out of the cavity.

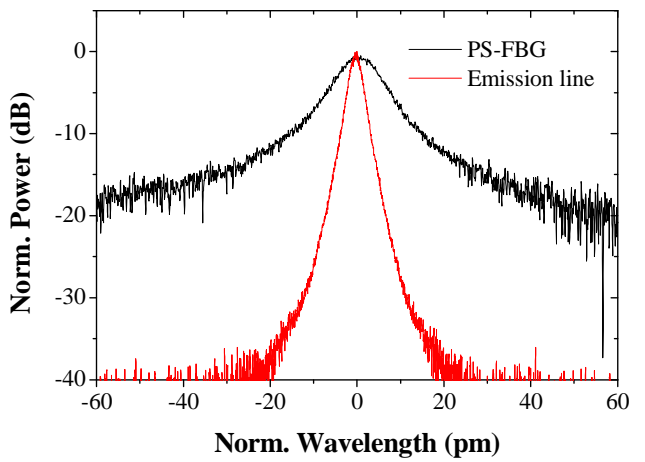

Fig. 10. Comparison between the emission lines generated when the filtering process takes place inside (red) and outside the laser's cavity (black).

\section{B. Multi-line emission.}

As previously stated, a significant feature of random lasers based on Raman scattering is its mode-less behavior. This particularity implies that there is no mode-hopping or modecompetition. Consequently the wavelength and peak power instabilities are smaller than in other fiber lasers as in erbium doped fiber-based lasers. Moreover, there is little or negligible gain competition between emission lines when operating a multi-wavelength fiber laser with the filtering devices placed appropriately [20, 27]. In order to exploit these advantages the setup reported in section 2.1 has been adapted to a fourwavelength laser with narrow line-widths.

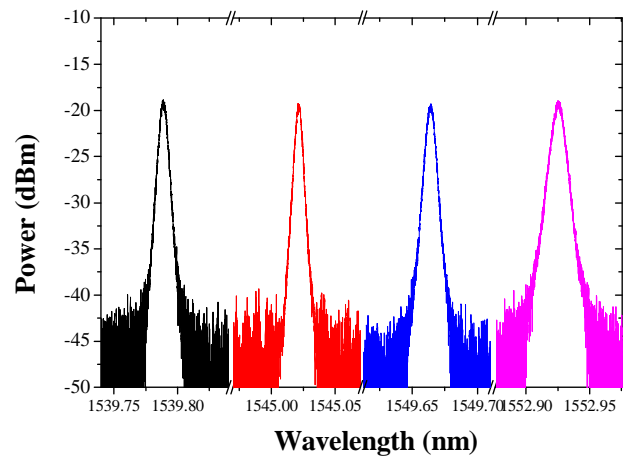

Fig. 11. Spectra of the four emission lines measured at the BOSA with a $0.08 \mathrm{pm}$ resolution.

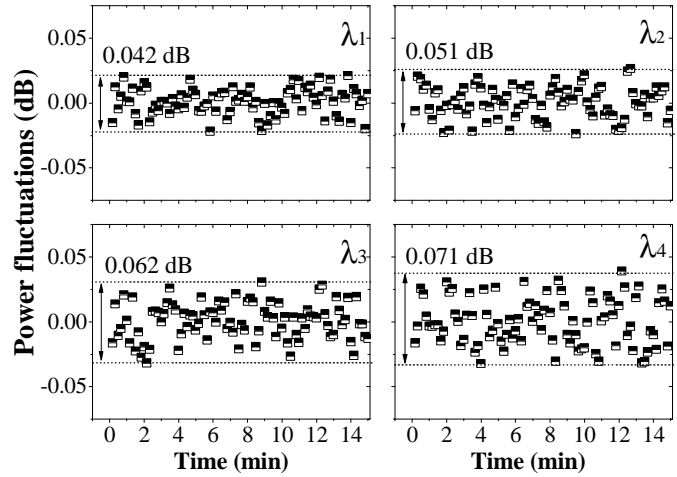

Fig. 12. Power instability of the four emission lines in 15 minutes.

The spectrum of the four emission lines can be seen in Fig. 11. Note that the wavelength axis has been clipped in order to allow the line-widths to be properly displayed. In this case a $90 / 10$ coupler was used for the measurements, extracting a $10 \%$ of the power through the output port. Therefore, low peak power values are attained in agreement with the case of $90 \%$ of recirculated power shown in Fig. 6. There are also some extra losses with respect to the single-line setup due to the inclusion of components. Accordingly the lasing thresholds are higher in this case, being 1.4, 1.42, 1.44 and $1.47 \mathrm{~W}$ for the first, second, third and fourth emission line respectively. The spectral line-widths of the four emission lines placed at the wavelengths 1539.8, 1545, 1549.6 and $1552.9 \mathrm{~nm}$ are 5.74, 5.04, 6.36 and $9.16 \mathrm{pm}$ respectively, which are greater than the obtained for a single emission line. A reason for this increase is that each PS-FBG has a different 
spectral response, being this particularly evident in $\lambda_{4}$. Additionally, since the required pump power is in this case much higher than the individual lasing threshold, an increase of the line-width due to broadening non-linear effects occurs (as previously shown in Fig. 5 for a single line).

The inhomogeneous behavior of the Raman gain permits the emission lines to be adjusted independently [27], in contrast for instance with EDFLs, where the variation of one emission line unavoidably affects the gain provided to the other lines[5,28]. In Fig. 11 the multi-wavelength laser was easily equalized by means of variable attenuators to achieve identical peak power in each emission line. This lack of gain competition together with the absence of mode-hopping and mode-competition result in low power instability over time. Fig. 12 shows the peak power fluctuations of the four emission lines every 10 seconds for 15 minutes (pump power $=1.48$ W). The instabilities attained for $\lambda_{1}$ to $\lambda_{4}$ are $0.042,0.051$, 0.062 and $0.071 \mathrm{~dB}$ respectively. It is worth mentioning that power and wavelength stabilities are affected by temperature variations. This is due to the wavelength dependence of the PS-FBG on the temperature. In addition, the gain provided by the Raman scattering effect also varies with temperature. Accordingly, these results may possibly be improved by better isolating the system.

\section{Discussion}

Previous published works about narrow random lasers use Fabry Perot filters, Mach-Zehnder interferometers and diverse types of gratings to obtain a different number of emission lines with line-widths of tens of picometers [18,19,21]. Other studies have also shown that a random DFB fiber laser spectrum can be modified at will by using programmable filters [22] appropriately placed in order to avoid or enhance power redistribution between lines [20]. A direct conclusion of this customizability is that narrower random DFB fiber lasers could be straightforwardly achieved if more selective filtering devices are used, always taking into account the contribution of broadening effects, which should be further studied. But for the first time, sub-gigahertz random laser emission lines have been reached in this work, allowing the direct real-time study of the statistical and temporal properties of DFB random lasers [25].

In addition, random DFB fiber lasers have interesting properties for sensing applications as reported in [11-14] due to its good performance in terms of tunability and wavelength operation. Moreover, high power and wavelength stabilities together with narrow emission lines could give precise amplitude and wavelength-based measurements if FBGs are used for the wavelength selection. This dependence of the Bragg wavelength on temperature and strain can be also utilized to control the wavelength of the emission line in the proposed setups. Moreover, extra emission lines could be generated using the multi-wavelength scheme just by increasing the number of channels of the MUX/DEMUX and including the corresponding Bragg gratings. Consequently, with an appropriate control of the Bragg wavelengths, each emission line of the multi-wavelength scheme could be independently tuned in both amplitude and wavelength (within the MUX/DEMUX channel bandwidth). This versatility is of particular interest when compared to Fabry Perot filter-based systems, since FFP filters do not allow each line to be adjusted independently.

\section{CONCLUSION}

In this work, sub-gigahertz line-widths have been reached for the first time in a random distributed feedback laser. Two random DFB fiber laser schemes based on a simple and customizable configuration have been reported. The gain is provided by the Raman scattering effect along $50 \mathrm{~km}$ of single-mode fiber which also act as a distributed mirror. The filtering process is realized by fiber Bragg gratings placed in a loop mirror at the end of the cavity. In the first topology a single emission line is attained with a line-width of $3.2 \mathrm{pm}$ which improves significantly (approx. 10 times) any other published work up to date. A second scheme is also presented for multi-wavelength operation where four narrow emission lines have been obtained with line-widths between 5 and $9 \mathrm{pm}$. This topology can be extended for a greater number of lines and presents a good versatility since the wavelength and peak power of each emission line can be independently adjusted.

\section{REFERENCES}

[1] V. Letokhov, "Stimulated emission of an ensemble of scattering particles with negative absorption," JETP Lett. 5,pp. 212-215, 1967.

[2] D. S. Wiersma, "The physics and applications of random lasers," Nat. Phys. Vol. 4, no. 5, pp. 359-367, 2008.

[3] S. Turitsyn, S. Babin, A. E. El-Taher, P. Harper, D. V. Churkin, S. I. Kablukov, J. D. Ania-Castañón, V. Karalekas, and E. V. Podivilov, "Random distributed feedback fibre laser," Nat. Photon., vol. 4, no. 4, pp. 231-235, 2010.

[4] J. Nuño del Campo, M. Alcon-Camas, and J. D. Ania-Castañón, "RIN transfer in random distributed feedback fiber lasers", in Advanced Photonics Congress (OSA), p. JM5A.7, 2012.

[5] X.-H. Jia, Y.-J. Rao, F. Peng, Z.-N. Wang, W.-L. Zhang, H.-J. Wu, and Y. Jiang, "Random-lasing-based distributed fiber-optic amplification," Opt. Exp., vol. 21, no. 5, pp. 6572-6577, 2013.

[6] S. A. Babin, A. E. El-Taher, P. Harper, E. V. Podivilov, and S. K. Turitsyn, "Tunable random fiber laser," Phys. Rev. A, vol. 84, no 2, pp. 231, 2011.

[7] M. Bravo, M. Fernandez-Vallejo, and M. Lopez-Amo , " Internal modulation of a random fiber laser," Opt. Lett., vol. 38, no. 9, pp. 15421544, 2013.

[8] I. D. Vatnik, D. V. Churkin, S. A. Babin, and S. K. Turitsyn, "Cascaded random distributed feedback Raman fiber laser operating at $1.2 \mu \mathrm{m}$," Opt. Exp., vol. 19, no. 19, pp. 18486-18494, 2011.

[9] W. L. Zhang, Y. J. Rao, J. M. Zhu, Z. X. Yang, Z. N. Wang, and X. H. Jia, "Low threshold 2nd-order random lasing of a fiber laser with a halfopened cavity," Opt. Expr., vol. 20, no. 13, pp. 14400-14405, 2012.

[10] S. A. Babin, I. D. Vatnik, A. Yu. Laptev, M. M. Bubnov, and E. M. Dianov, "High-efficiency cascaded Raman fiber laser with random distributed feedback," Opt. Exp., vol. 22, no. 21, pp. 24929-24934, 2014.

[11] Z. N. Wang, Y. J. Rao, H. Wu, P. Y. Li, Y. Jiang, X. H. Jia, and W. L. Zhang, "Long-distance fiber-optic point-sensing systems based on random fiber lasers," Opt. Expr., vol. 20, no. 16, pp. 17695-17700, 2012.

[12] M. Bravo, V. DeMiguel-Soto, A. Ortigosa and M. Lopez-Amo, "Fully switchable multi-wavelength fiber laser based interrogator system for remote and versatile fiber optic sensors multiplexing structures" Proc. SPIE, 9157, 91576, 2014.

[13] A. M. R. Pinto, M. Lopez-Amo, J. Kobelke, and K. Schuster, "Temperature fiber laser sensor based on a hybrid cavity and a random mirror," J. Lightwave Technol., vol. 30, no. 8, pp. 1168-1172, 2012.

[14] X. H. Jia, Y. J. Rao, Z. N. Wang, W. L. Zhang, Y. Jiang, J. M. Zhu, and Z. X. Yang, "Towards fully distributed amplification and highperformance long-range distributed sensing based on random fiber laser," Proc. SPIE 8421, 842127, 842127-4, 2012.

[15] M. Fernandez-Vallejo, S. Rota-Rodrigo, and M. Lopez-Amo, "Comparative study of ring and random cavities for fiber lasers," Appl. Opt., vol. 53, no. 16, pp. 3501-3507, 2014. 
[16] A. M. R. Pinto, M. Bravo, M. Fernandez-Vallejo, M. Lopez-Amo, J. Kobelke, and K. Schuster, "Suspended-core fiber Sagnac combined dual-random mirror Raman fiber laser," Opt. Expr., vol. 19, no. 12, pp. 11906-11515, 2011.

[17] A.M.R. Pinto, O. Frazão, J.L. Santos, M. Lopez-Amo, "Multiwavelength Raman Fiber Lasers Using Hi-Bi Photonic Crystal Fiber Loop Mirrors Combined With Random Cavities," J. Lightwave Technol, vol. 29, no. 10, pp.1482-1488, 2011.

[18] S. Sugavanam, N. Tarasov, X. Shu, and D. V. Churkin, "Narrow-band generation in random distributed feedback fiber laser," Opt. Exp. vol. 21, no. 14, pp.16466-16472, 2013.

[19] L. Wang, X. Dong, P. P. Shum, and H. Su, "Tunable Erbium-Doped Fiber Laser Based on Random Distributed Feedback," IEEE Photon. Journal, vol. 6, no. 5, art. no. 6884783, 2014.

[20] S. Sugavanam, Z. Yan, V. Kamynin, A. S. Kurkov, L. Zhang, and D. V. Churkin, "Multiwavelength generation in a random distributed feedback fiber laser using an all fiber Lyot filter," Opt. Expr., vol. 22, no. 3, pp. 2839-2844, 2014.

[21] Y. Zhu, W. Zhang, and Y. Jiang, "Tunable multi-wavelength fiber laser based on random Rayleigh backscattering," IEEE Photon. Technol. Lett., vol. 25, no. 16, pp. 1559-1561, 2013.

[22] V. DeMiguel-Soto, M. Bravo M, and M. Lopez-Amo, "Fully switchable multiwavelength fiber laser assisted by a random mirror," Opt. Lett. vol. 39, no. 7, pp. 2020-2023, 2014.

[23] S. K. Turitsyn, S. A. Babin, D. V. Churkin, I. D. Vatnik, M. Nikulin, and E. V. Podivilov, "Random distributed feedback fibre lasers," Phys. Reports, vol. 542, pp. 133-193, 2014.

[24] D. Leandro, R.A. Perez-Herrera, I. Iturri, M. Lopez-Amo, "Experimental study of the SLM behavior and remote sensing applications of a multiwavelength fiber laser topology based on DWDMs," Applied Physics B: Lasers and Optics, vol. 118, no. 3, pp. 497-503, 2015.

[25] O.A. Gorbunov, S. Sugavanam, and D. V. Churkin, "Intensity dynamics and statistical properties of random distributed feedback fiber laser," Opt. Lett. vol. 40, no. 8, pp.1783-1786, 2015.

[26] D.V. Churkin, I.V. Kolokolov, E.V. Podivilov, I.D. Vatnik, M.A Nikulin, S.S. Vergeles, I.S. Terekhov, V.V. Lebedev, G. Falkovich, S.A Babin, S.K. Turitsyn, "Wave kinetics of random fibre lasers," Nat. Comm. vol. 6, no. 6214, 2015.

[27] A. E. El-Taher et al., "Dual-wavelength ultralong Raman laser with Rayleigh-scattering feedback," Opt. Lett., vol. 35, no. 7, pp. 1100, 2010.

[28] A. E. El-Taher, P. Harper, S. A. Babin, D. V. Churkin, E. V. Podivilov, J. D. Ania-Castanon, and S. K. Turitsyn, "Effect of Rayleigh-scattering distributed feedback on multiwavelength Raman fiber laser generation," Opt. Lett. vol. 36, pp.130-132, 2011.

Daniel Leandro was born in Pamplona, Spain, in November 1984. He received the telecommunication engineering and master degree from the Universidad Pública de Navarra, Spain in 2010 and 2012, respectively. In 2012, he joined the Optical Communications Group, Department of Electrical and Electronic Engineering from Universidad Publica de Navarra. He has been a visiting Ph.D. student at the School of Engineering and Mathematical Sciences at City University of London. His research interests are in fiber optic lasers, optical amplifiers, optical fiber sensor networks and sensor multiplexing architectures.

Sergio Rota-Rodrigo was born in Navarra, Spain, in October 1985. He received the Telecommunication Technical Engineering, Telecommunication Engineering and Communications Master degrees from the Universidad Pública de Navarra, Spain in 2007, 2010 and 2011, respectively. In 2011, he joined the Optical Communications Group, Department of Electrical and Electronic Engineering from Universidad Pública de Navarra. His research interests are in fiber optic lasers, optical amplifiers, optical fiber sensor networks, photonic crystal fibers and chemical fiber optic sensors.

Diego Ardanaz was born in Pamplona, Spain, in May 1988. He received the telecommunication engineering degree from the Universidad Publica de Navarra, Spain in 2015. During 2014 and 2015 he worked in the Optical Communications Group, Department of Electrical and Electronic Engineering from Universidad Publica de Navarra. His research interests are in fiber Bragg gratings, fiber lasers, optical fiber sensor networks and multiplexing architectures.
Manuel Lopez-Amo (M'91, SM '98) is a full professor in photonics at the Electrical and Electronic department of Universidad Pública de Navarra (Spain) since 1996. He is the head of the optical communications group of this department. He has been Chairman of the Optoelectronic Committee of Spain. He has been the director of the Engineering Faculty and of the Electrical and Electronic department of Universidad Pública de Navarra. He has been the leader of more than fifty research projects and he has coauthored more than 250 works in international refereed journals and conferences related with fiber-optic networks, fiber-optic sensors, fiber-optic amplifiers and lasers and integrated optics. He has supervised fifteen PhD theses about Photonics. He is a member of more than twenty international Committees of Conferences, being the technical chair of the last International Conference on fiber optic sensors (OFS 2014). 